Geoffrey C. Clayton and Peter G. Martin

Department of Astronomy, University of Toronto

\title{
ABSTRACT
}

New IUE observations confirm the differences between the Galactic and LMC ultraviolet extinction curves and show some evidence for variations within the LMC. Visual and infrared photometry and polarimetry show that the anomalous properties of the LMC dust do not extend to longer wavelengths. Despite the much different dust to gas ratios in the Galaxy and the Magellanic clouds, the dust formation efficiency is similar when the abundances are included.

\section{EXTINCTION AND POLARIZATION}

Significantly reddened stars are rare in the LMC but extinction curves were constructed for 12 stars from 0.13 to $2.2 \mu \mathrm{m}$. The wavelength dependence of interstellar linear polarization was studied from 0.35 to $0.84 \mu \mathrm{m}$ for 18 stars.

The differences in the average extinction curves of the two galaxies, seen in the ultraviolet, are confirmed in the present study. In the visual and infrared the curves are very similar. The polarization characteristics are also very similar. Typically, the Serkowski curve can be fit to the LMC data with the same parameters used to fit Galactic polarization (clayton, Martin, and Thompson 1983). The polarization efficiency is also comparable.

Apparently the relevant variables, namely grain size, shape and composition, magnetic field strength, and gas density and temperature all conspire to make the grain alignment and polarization efficiency the same in these two quite different galaxies. The similarities between the extinction and polarization properties of interstellar dust in the LMC and the Galaxy are more remarkable than the differences considering the large number of potentially free parameters involved in modelling the dust. 
2. VARIATION OF EXTINCTION WITH LOCATION IN THE LMC

The extinction curves measured for individual lines of sight within the Galaxy are often quite different from the average curve. Similar variations were searched for in the LMC data but any variations present are no larger than the estimated errors. To reduce these errors, the stars were grouped into 2 bins; one containing stars with a projected distance from the centre of 30 Dor of less than one degree and the other containing those lying at a distance greater than one degree. The average extinction curves for the 2 bins are different. The 30 Dor hin has a smaller $2200 \AA$ blump and higher far ultraviolet extinction. However, these differences are only marginally significant.

If these regional differences are real, then it is possible that the grains near 30 Dor, a highly active region, have been modified. One possibllity is sputtering and grain-grain collisions caused by shock waves. Massa and Savage (1983) have shown that a possible result of shocks is a steepening of the far ultraviolet extinction and a smaller $2200 \AA$ bump. This is the trend seen in the binned data.

\section{ABUNDANCES AND THE DUST-TO-GAS RATIO}

The dust-to-gas ratio, $E(B-V) / N_{H}$, in the LMC is several times lower than the Galactic value (Koornneef 1982) which could be interpreted as evidence of less efficient dust production in the LMC. However, the amount of dust produced is 1 imited by the abundance of condensable species. Taking into account the lower CNO abundances found for the LMC (Dufour et al. 1982), the dust to gas ratio, $E(B-V) / N_{C N O}$, is within a factor of two of the galactic value. For the SMC, which has even lower CNO abundances and $\mathrm{E}(\mathrm{B}-\mathrm{V}) / \mathrm{N}_{\mathrm{H}}$ of one-tenth the Galactic value, the ratio $\mathrm{E}(\mathrm{B}-\mathrm{V}) / \mathrm{N}_{\mathrm{CNO}}$ is in the same range as the LMC and the Galaxy. Therefore, it can be concluded that the dust formation efficiency is rather similar in these 3 galaxies.

It is interesting that the average extinction curve in the SMC is even more extreme than the IMC curve: the far ultraviolet extinction is higher and the bump is almost non-existent (Bromage and Nandy 1983). Therefore, at least for this small sample of 3 galaxies, there is an apparent correlation between the CNO abundances and the ultraviolet extinction characteristics.

\section{REFERENCES}

Bromage, G.E., and Nandy, K. 1983, Monthly Notices Roy. Astron. Soc. 204, pp. 29p.

Clayton, G.C., Martin, P.G., and Thompson, I. 1983, Astrophys. J. 265, 194 Dufour, R.J., Shields, G.A., and Talbot, R.J. 1982, Astrophys. J. 252, pp. 461 .

Koornneef, J. 1982, Astron. Astrophys. 107, 247

Massa, D., and Savage, B.D. 1983, preprint. 